\title{
Etude socio-économique et technologique de la production du poisson fermenté et séché (Guedj) au Sénégal
}

\author{
N. G. FALL ${ }^{1,3^{*}}$, L.T. TOUNKARA ${ }^{1}$, M.B. DIOP ${ }^{2}$, O.T. THIAW ${ }^{3}$ et P. THONART $^{4}$ \\ ${ }^{1}$ Institut de Technologie Alimentaire (ITA) BP : 2765 Hann-Dakar, Sénégal. \\ ${ }^{2}$ Université Gaston Berger, Unité de Formation et de Recherche des Sciences Agronomiques de l'Aquaculture \\ et des Technologies Alimentaires (UFR S2ATA), BP 234 Saint-Louis, Sénégal. \\ ${ }^{3}$ Université Cheikh Anta DIOP, Institut Universitaire de Pêche et d'Aquaculture (IUPA) de Dakar (UCAD), \\ BP : 45784 Dakar-Fann, Sénégal. \\ ${ }^{4}$ Université de Liège Gembloux Agro Bio Tech, Centre Wallon de Biologie Industrielle (CWBI), \\ Passage des Déportés, 2 B-5030 Gembloux, Belgique. \\ *Auteur correspondant, E-mail: nba@ita.sn
}

\section{RESUME}

Cette étude aborde les aspects socio-économiques et technologiques relatifs à l'un des maillons très important de la pêche maritime qui est la transformation du poisson en guedj ou poisson fermenté et séché. En effet, une enquête diagnostique menée à travers trois zones de production au Sénégal (Dakar, Thiès et Fatick), combinée aux données de la bibliographie et des entretiens avec les acteurs, ont permis de faire ressortir un certain nombre d'enjeux. L'étude a montré que cette activité assurée à $80 \%$ par des femmes, génère beaucoup d'emplois et de revenus, que le prix de vente fluctue entre 1000 et 3500 frs CFA. $\mathrm{kg}^{-1}$ et par conséquent qu'une grande productrice peut gagner en moyenne jusqu'à 400000 frs CFA par mois. Cependant, malgré son importance économique, elle est confrontée à d'énormes difficultés liées à la manutention, au manque d'infrastructures et de stockage adéquates et aux techniques de transformation rudimentaires utilisées. Par ailleurs, l'analyse physicochimique et microbiologique réalisée sur des échantillons prélevés dans les sites de production et de vente laisse apparaître la nécessité d'appliquer rigoureusement les bonnes pratiques de fabrication et d'hygiène afin d'améliorer la qualité sanitaire du guedj, qui fait de plus en plus l'objet de demande du marché extérieur.

(C) 2014 International Formulae Group. All rights reserved.

Mots clés : Guedj, poisson fermenté, méthode traditionnelle, étude de filière, qualité, Sénégal.

\section{INTRODUCTION}

Le Sénégal est connu pour son niveau élevé de production de produits de mer. La moyenne des débarquements de la pêche maritime sur la période 2006-2011 a été de 377199 tonnes dont $89 \%$ assurée par la pêche artisanale (DPM, 2006-2011). Mais les contraintes socio-économiques entravent le développement de la conservation industrielle des captures (Die-Ouadi, 2005). Des pertes post captures énormes sont déplorées alors que paradoxalement la ressource se raréfie de plus en plus. C'est ainsi la transformation artisanale s'impose comme seule moyen de 
conservation relativement simple aux surplus des débarquements invendus et aux déchets. C'est une forme de valorisation de produits halieutiques la plus ancienne. $40 \%$ des débarquements de la pêche artisanale vont à la transformation traditionnelle (Mbaye, 2005). Elle représente un segment non négligeable pour l'amélioration des conditions de vie des populations, surtout celles rurales en termes d'emploi, de revenus et de contribution à l'atteinte de la sécurité alimentaire.

La quantité de produits transformés en 2011 était estimée à 49881 tonnes, soit une Valeur Commerciale Estimative de 21512 951 (VCE*1000) (DPM, 2011). Ils font partie intégrante des habitudes culinaires des sénégalais et par conséquent, contribuent à la satisfaction de la demande en protéine (Mbaye, 2005). Il s'agit de produits braisés séchés, salés séchés, fumés et fermentés séchés. Ces derniers sont estimés en 2011 à 4 551 tonnes (soit 9,12\% de la production annuelle) pour une Valeur Commerciale Estimative de 1962780 F CFA (DPM, 2011). Ils sont couramment rencontrés en Afrique sous plusieurs noms tels que (momoni, koobi et kako au Ghana, lanhouin au Bénin et au Togo, adjuevan en Côte d'Ivoire et guedj au Sénégal) (Kouakou et al., 2013) et représentent la majorité des produits transformés de la pêche artisanale en Asie du Sud Est dont plaa-soom de la Thaïlande (Kopermsub et Yunchalard, 2010) ou Nuocmam du Vietnam (Hubert, 2003). Ces derniers sont aujourd'hui des sauces condimentaires familières en Europe (Hubert et Sabban, 2005).

Le guedj ou poisson fermenté au Sénégal est obtenu par une fermentation spontanée comme le cas du lanhouin (Anihouvi et al., 2005). C'est un produit semi séché brun clair et caractérisé par une forte odeur âcre. Il sert à la fois d'aliment et de condiment ajouté en petite quantité aux ragoûts et soupes pour donner du goût aux préparations culinaires. Ce produit présente des atouts économiques majeurs qui méritent beaucoup plus d'attention. Cependant, la nature artisanale de sa production pose des problèmes liés à la santé, par la présence éventuelle de substances toxiques pouvant affecter la qualité organoleptique et sanitaire du produit.

Le but de cette étude est de cerner les contraintes majeures qui compromettent le développement de ce secteur et ceci à travers des enquêtes réalisées auprès des acteurs et une caractérisation chimique et microbiologique du produit fini.

\section{MATERIEL ET METHODES}

\section{Site d'étude}

Les régions de Dakar, Thiès et Fatick ont été sélectionnées comme zones d'études et leur choix a été guidé par le fait que $54 \%$ du guedj produit provient de ces régions (DPM, 2011). En outre, la majorité des acteurs de la transformation artisanale des produits halieutiques habitent dans ces zones qui abritent les plus grandes unités de production, les quais de pêche et les marchés d'écoulement de toutes sortes de produits transformés à l'état frais ou sec.

\section{Matériel biologique}

Il est constitué de guedj fabriqué à partir de trois espèces de poissons gras (mâchoiron ou Arius latisculatus), moyennement gras (capitaine ou Pseudotolithus brachygnatus) et maigres (sompate ou Pomadasys jubelini).

\section{Méthodologie}

Deux types de questionnaire ont été élaborés et formulés sur la base d'informations issues de la littérature et des 
entretiens avec quelques acteurs de la filière: le premier portant sur la production a permis de recueillir des données sur les procédés de transformation (identification des acteurs, description de l'activité, source et évolution de l'approvisionnement, types d'espèces utilisées, critères de sélection des espèces, quantité de produit traitée par jour, équipements et emballages utilisés) et le second a abordé les aspects liés à la commercialisation et à la consommation des produits transformés (prix, marché d'écoulement, clientèle, revenu mensuel, nombre de personnes dépendantes, problèmes rencontrés, préférence). Dans l'ensemble, 50 producteurs, 20 commerçants et 20 consommateurs ont été contactés. Les visites ont concerné 9 sites de productions et 6 marchés.

Les paramètres microbiologiques et chimiques ont été évalués à partir d'échantillons de guedj collectés dans des sites de transformation et de vente. Au total, 19 échantillons de guedj fabriqués à partir des trois espèces citées ci-dessus ont été caractérisés. La flore aérobie totale, les coliformes fécaux, les clostriduim sulfitoréducteurs, les staphylocoques pathogènes, les salmonelles, les lactobacillus ont été recherchés suivant les normes NF (ISO 4833, V08060, EN ISO 6888-1, V08-052) et XP V08-061. L'humidité, les matières grasses, les protéines et les sels ont été déterminés respectivement par les méthodes 945.15, 2003.06, 2001.11 et 937.09 d'AOAC (2007). L'histamine est déterminée par HPLC et le $\mathrm{pH}$ lu grâce à un pH-mètre de marque Oakton, Acorn ${ }^{\mathrm{TM}}$ meter Kit.

\section{Analyses statistiques}

Les données obtenues à partir de l'enquête, de la base de données de la Direction des Pêches Maritimes (DPM) et des caractérisations chimiques et microbiologiques du produit fini ont été analysées puis traitées avec Microsoft Office Excel 2007.

\section{RESULTATS}

\section{Situation géographique}

Le guedj est produit sur toute la façade maritime et dans certains estuaires du Sénégal. Les plus grandes unités de production sont localisées dans le voisinage des principales zones de débarquements à travers le pays notamment dans les régions de Thiès (Mbour, Kayar, Fass boye), de Dakar (Pikine, Rufisque, Bargny), de Ziguinchor (Kafountine) et de Fatick (Dionewar, Niodior, Falia, Djiffer), où vivent la majorité des pêcheurs et les transformateurs.

\section{Analyse fonctionnelle de la filière}

Les acteurs de la filière sont nombreux. Les pêcheurs assurent la quête de la matière première par la pêche et le livrent aux transformateurs. Ces derniers assurent toutes les opérations du système de production (éviscération, parage, salage, fermentation, séchage). La plus part d'entre eux sont des Sénégalais (90\%), (soit $80 \%$ de femmes et $10 \%$ d'hommes) contre $10 \%$ d'étrangers hommes ressortissants des pays comme (Mali, Guinée, Burkina Faso). La main d'œuvre est constituée de femmes et majoritairement d'hommes. Il est important de noter que les quelques hommes impliqués dans le système de transformation sont des employés ou sourguas des femmes transformatrices membres des coopératives et propriétaires des moyens de production. Ils assurent entres autres, les travaux de manutention, d'approvisionnement, de découpe, d'emballage, d'opérations de nettoyage et de désinfection des sites de transformation. Ils sont majoritairement des étrangers (Mali, 
Guinée, Burkina Faso) avec quelques rares Sénégalais. Les commerçants sont constitués de collecteurs ou grossistes et détaillants ou bana-banas. Ils proviennent des marchés locaux de l'intérieur du pays et même parfois de la sous-région. Ils se chargent de la vente en gros et/ou en détail du produit. Enfin les consommateurs sont à l'aval du circuit et sont les destinataires finaux dont la demande constitue un levier important et déterminant de l'activité de transformation.

\section{Analyse commerciale de la filière}

Le guedj obéit par excellence à la loi de l'offre et de la demande. Le prix de vente qui varie entre 1000 et 3000 frs CFA le kilogramme est fonction des saisons (prix élevés pendant l'hivernage), de la nature et la taille des espèces, des lieux de débarquement et enfin de la concurrence déloyale des collecteurs nationaux et/ou étrangers. L'offre globale du guedj est destinée d'une part au marché national contrôlé par des distributeurs locaux (bana-banas) ou détaillants, et d'autre part au marché extérieur géré essentiellement par des grossistes ou collecteurs. Au niveau national, les produits transformés sont commercialisés par ordre décroissant dans les régions de Dakar, Kolda, Diourbel, Kaolack, Thiès, Matam, Louga, Tambacounda, Ziguinchor, Fatick, Saint-Louis (DPM, 2011). L'essentiel des flux d'exportations de ces produits est orienté vers les pays de la sousrégion (Burkina Faso, Guinée Conakry, Mali, Côte d'Ivoire, Gambie, Togo, Guinée Bissau et Congo) (Figure 1) (DPM, 2011). En 2011, le plus grand importateur de guedj est le Mali, suivi de la Guinée-Conakry, du Congo, de la Gambie, du Togo et de la Guinée Bissau (Figure 2) (DPM, 2011). Notons que ces quantités ne tiennent pas compte des flux informels qui représentent la majorité des exportations des pays voisins. Les plus grandes destinations enregistrées en 2011 pour le marché européen sont l'Italie, la France et la Belgique. Ce marché, bien que regorgeant de nombreux ressortissants Africains et Asiatiques, n'est pas grand demandeur de ce produit malgré les nombreuses initiatives d'exportateurs privés. La qualité du produit constitue une entrave sérieuse à son accès à ce marché porteur.

\section{Analyse socio-économique de la filière}

Au Sénégal, les tendances au cours des années 2006 à 2011 montrent une nette progression de la transformation artisanale du poisson frais en divers produits dérivés qui varie selon le type, la qualité, la taille de l'espèce et la technologie de transformation (Tableau 1) (DPM, 2006-2011). Les débouchés les plus fréquents sont : le kéthiakh (poisson braisé, salé et séché), le guedj (poisson fermenté et séché), le tambadieng (petit poisson entier salé et séché), le métorah (poisson fumé et séché), le saly (poisson salé et séché), le yet, les coquillages (toufa, pagne, yokhos), les ailerons de requins et les crevettes séchées. Sur une production totale estimée à 273329 t entre 2006 et 2011, le guedj vient en deuxième place après le kéthiakh avec environ 24269 t soit 9\% de la quantité totale de produits transformés (Tableau 1) (DPM, 2006-2011). En 2011, les principales régions productrices de guedj par ordre décroissante sont : Thiès, Ziguinchor, Dakar, Saint Louis, Fatick et Louga (DPM, 2011) (Figure 3).

Considérée comme une activité de rente, la production du guedj procure des revenus annuels importants à ses acteurs. Ainsi, il a été difficile de connaître avec exactitude les revenus générés par les acteurs du fait de la variabilité des gains qui dépendent des lois du marché et de la réticence de certains d'entre eux. Mais, l'enquête a révélé une moyenne de 400000 
frs CFA/mois pour une grande opératrice. Il ressort de l'étude que plus de $70 \%$ des femmes ont une expérience de plus de 10 ans. En moyenne, chacune prend en charge des familles larges de 6 à 8 personnes. La quasitotalité des acteurs sont des analphabètes (75\% des pêcheurs, $80 \%$ des transformateurs et $56 \%$ des commerçants).

\section{Techniques de production du Guedj Espèces utilisées}

Les espèces couramment utilisées pour la fabrication du guedj sont présentées au Tableau 2. Selon les résultats de l'enquête, le capitaine et le machoiron sont les espèces les plus utilisées par les transformateurs (Figure 4). $80 \%$ des consommateurs apprécient le guedj pour sa saveur, mais préfèrent généralement celui issu des espèces de poisson comme le Galeoides decadactylus ou faux capitaine et le Pseudotolithus brachygnatus ou capitaine pour leur haute qualité organoleptique. Auparavant, le guedj était consommé uniquement par les ménages ruraux. De nos jours, avec la conjoncture actuelle, il est souvent utilisé comme substitut au poisson frais. D'après les résultats de l'enquête, $93 \%$ des ménages enquêtés consomment cette denrée.

\section{Différentes formes de guedj}

Plusieurs types de guedj qui varient suivant les espèces et les localités ont été observés sur le marché en allant des gros aux petits poissons fermentés séchés. Ils sont découpés en portefeuille ou en darne. On peut citer entre autres: le guedj kong, le guedj yass, le guedj sick et le touboulane. La Figure 5 présente les formes de guedj issues de différentes espèces de poisson et dans plusieurs localités.

\section{Diagramme de production}

La technique de production du poisson fermenté et séché décrite par les personnes interviewées est presque identique. Deux méthodes de fabrication ont été largement décrites par les transformateurs comme méthodes couramment utilisées: la fermentation des petits poissons entiers avec salage à sec et séchage et la fermentation des gros poissons ouvert en portefeuille avec salage en saumure et séchage. Le procédé de fabrication du Guedj comprend plusieurs étapes présentées à la Figure 6.

\section{Approvisionnement en matière première}

Une fois débarqué, le poisson est mis à même le sol et transporté dans de petites camionnettes ou charrettes vers les lieux de production.

\section{Parage/lavage}

Le poisson subit un parage préliminaire (écaillage, éviscération, découpe...) à l'aide d'un couteau. Les gros poissons sont ouvert en portefeuille et les petits poissons sont laissées entiers avant d'être lavés. Le lavage se fait soit avec l'eau de mer ou l'eau de robinet.

\section{Salage}

Le salage est fait avec du sel marin en gros grains ou mixte (mélange de gros et petits grains). Il se fait soit en saumure pour les espèces gras ou à sec pour les espèces maigres. Le sel varie entre 4,9 et $9,6 \%$ selon les espèces. Dans le cas du salage à sec, le sel est appliqué directement à la surface du poisson, dans les branchies et sous l'opercule par frottement ou saupoudrage. Le salage par saumurage consiste à immerger le poisson dans une solution aqueuse de sel.

\section{Fermentation}

La fermentation dure 2 à 4 jours et varie en fonction du type, de la quantité et de la taille du poisson. Elle se réalise dans différents types de matériels (bacs ou cuves en 
ciment, bidons, bassines, jarres...). Elle est spontanée et consiste à une simple incubation des poissons crus additionnés de sel à des températures optimales pouvant atteindre ou dépasser $30^{\circ} \mathrm{C}$.

\section{Séchage}

Il est fait de manière traditionnelle sur des claies en bois de rônier, des filets de récupération, des nattes, des herbes ou à même le sol et à ciel ouvert. Le poisson est mis à sécher pendant 2 à 4 jours suivant les deux méthodes. Il est retourné une fois par jour (à la mi-journée), empilé et couvert de bâches en plastiques maintenues par des pierres avant le coucher du soleil.

\section{Conditionnement et emballage}

Après séchage, le guedj est conditionné et emballé dans du papier, des paniers, des sacs en toile ou en jute, des cartons, des caisses fermées avec des cordes et polypropylène. Ces matériaux sont utilisés soit pour l'entreposage et au cours de la distribution.

\section{Analyse Physico-chimiques \\ et microbiologiques du guedj \\ Les résultats des analyses physico-} chimiques et microbiologiques réalisées sur des échantillons de guedj collectés dans des marchés locaux et au niveau des sites de transformations et de vente sont résumés dans le Tableau 3. Le $\mathrm{pH}$ des échantillons varie entre 6,05-7,2. La teneur en eau de la majorité des échantillons est supérieure à la norme. La moyenne des résultats obtenus est de 50,35\% pendant que la teneur en sel varie entre 4,9 et $9,6 \%$. Le taux d'histamine mesuré reste faible pour toutes les espèces. Sur le plan nutritionnel, la teneur en protéines de la plupart des produits varie en moyenne entre 28 et $30 \%$ comparé au poisson frais dont la teneur varie entre 15 et $20 \%$. L'analyse microbiologique montre la présence élevée de la flore aérobie totale avec une moyenne de $18,4710^{4}$ ufc. ${ }^{-1}$. En revanche, aucune salmonelle n'a été identifiée sur les échantillons analysés. Mais on note la présence de clostridies dans certains échantillons avec une moyenne de 17,64 10 1 ufc. $g^{-1}$.

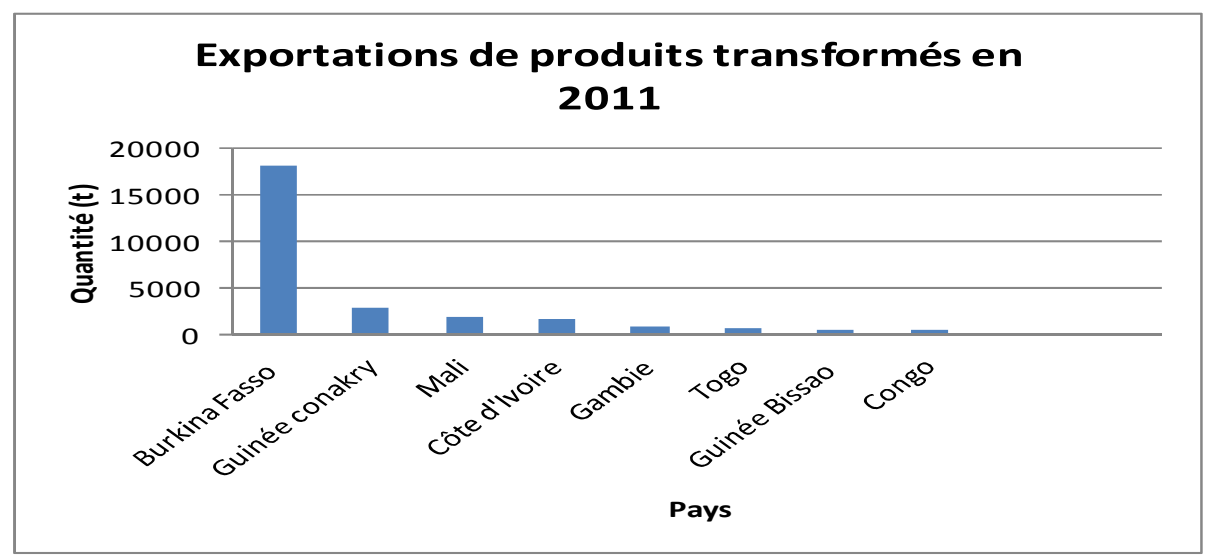

Figure 1 : Exportation de produits transformés en 2011 par pays. Source: Résultats généraux des pêches DPM, 2011. 


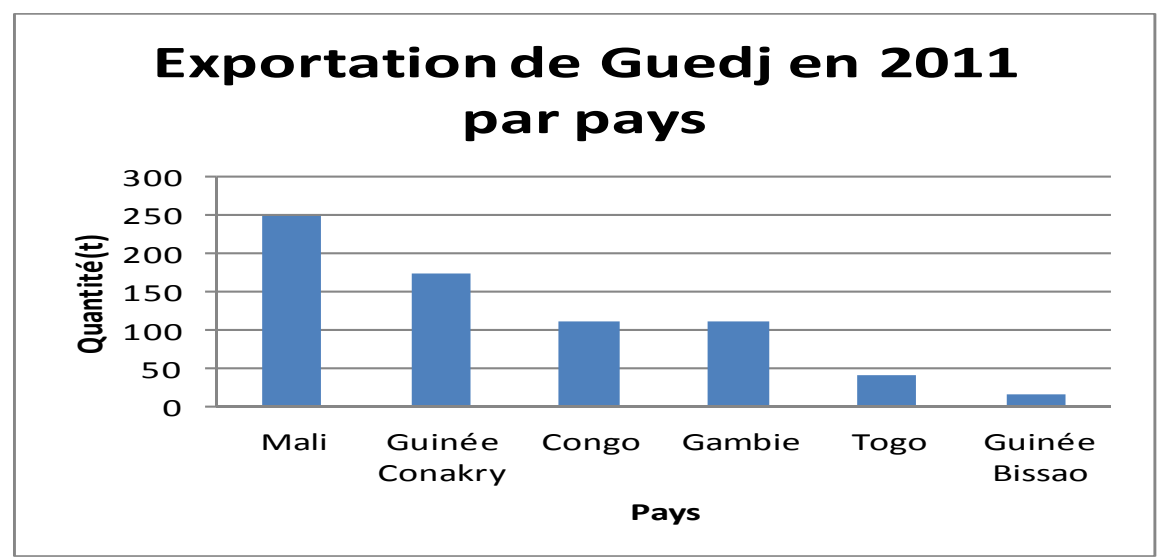

Figure 2 : Exportation de guedj en 2011 par pays. Source: Résultats généraux des pêches DPM, 2011.

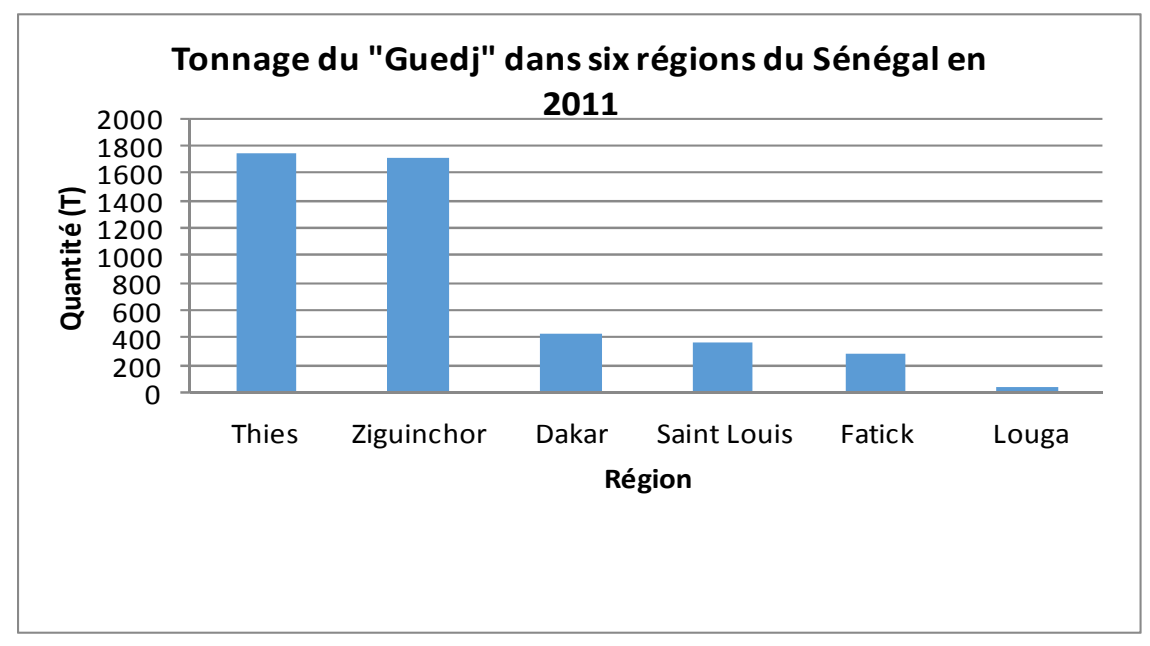

Figure 3 : Tonnage du guedj dans six régions du Sénégal en 2011. Source: DPM, 2011, Résultats généraux des pêches. 


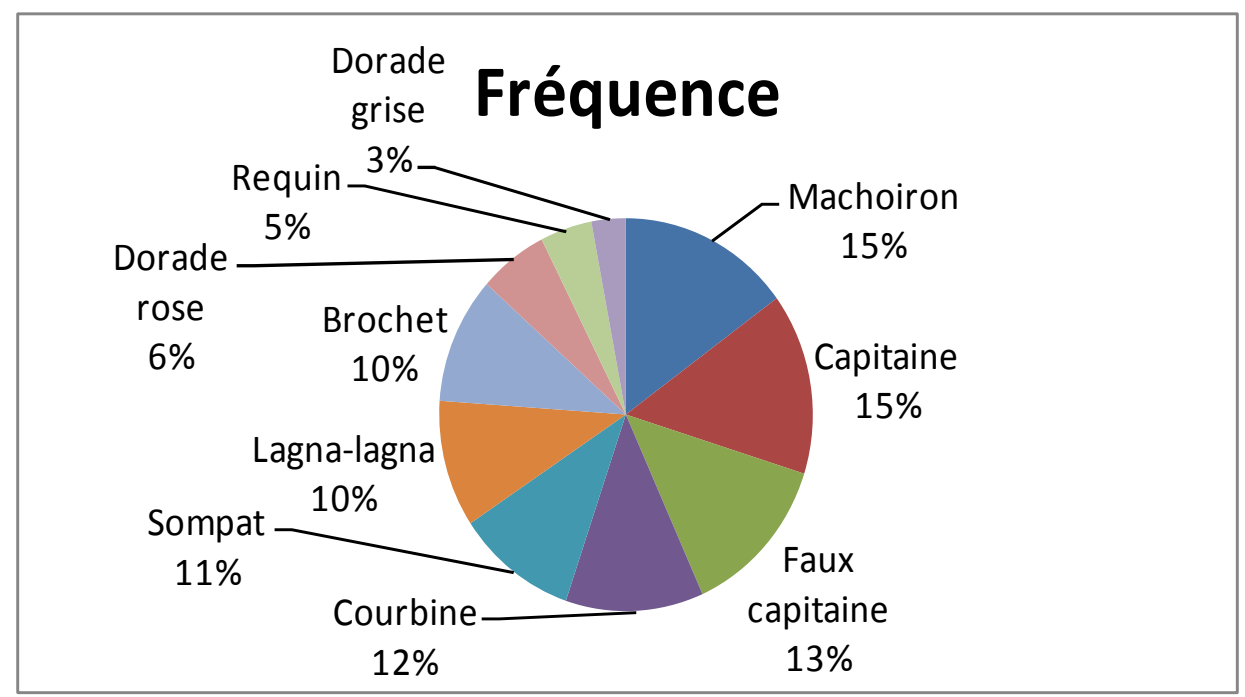

Figure 4 : Fréquence d'espèces utilisées pour la fabrication du guedj.
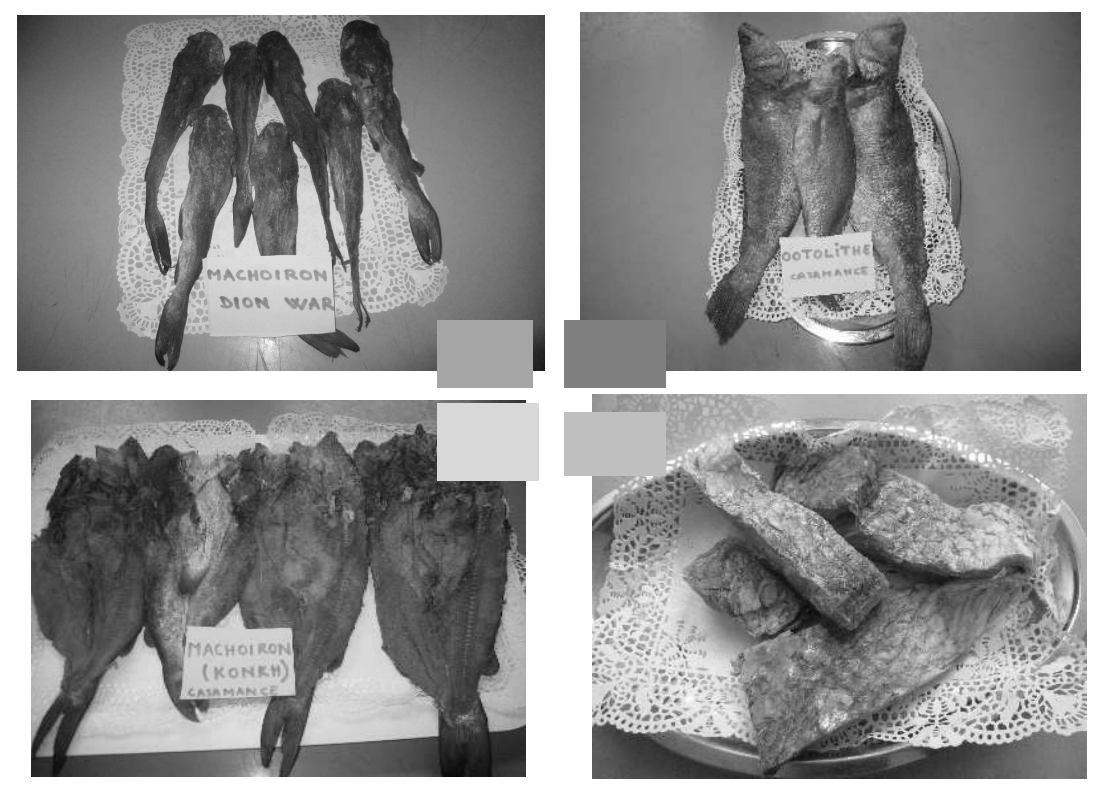

Figure 5 : Différentes formes de guedj. (a) et $(\mathrm{b}=$ entier ; (c) = découpe en portefeuille ; (d) = découpe en darne. 


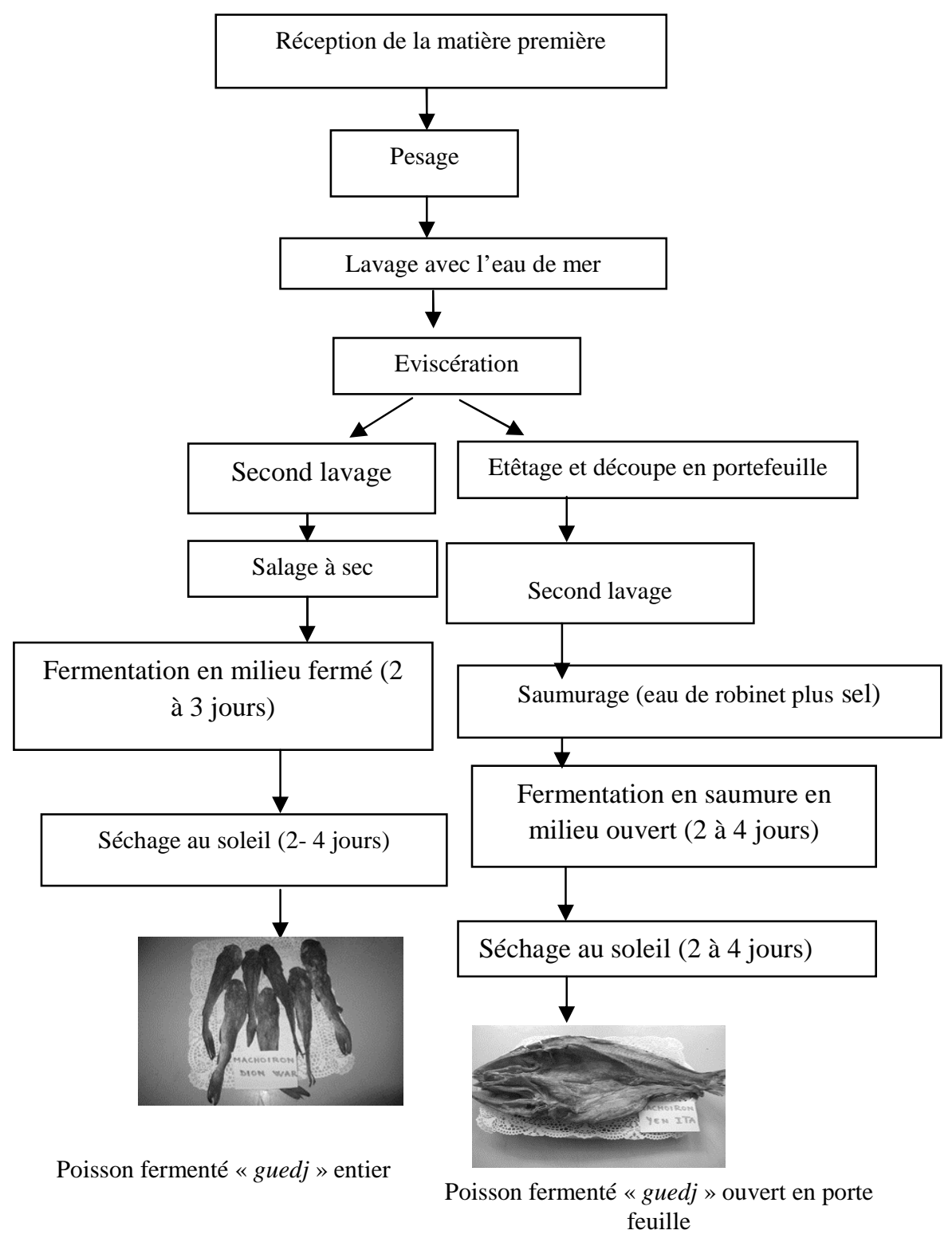

Figure 6 : Diagramme de fabrication du «guedj ». 
N. G. FALL et al. / Int. J. Biol. Chem. Sci. 8(6): 2523-2538, 2014

Tableau 1 : Quantité annuelle (en tonnes) de 2006 à 2011 des produits dérivés de la transformation artisanale Sénégal.

\begin{tabular}{lccccccc}
\hline \multirow{2}{*}{ Produits } & \multicolumn{7}{c}{ Année } \\
\cline { 2 - 7 } & $\mathbf{2 0 0 6}$ & $\mathbf{2 0 0 7}$ & $\mathbf{2 0 0 8}$ & $\mathbf{2 0 0 9}$ & $\mathbf{2 0 1 0}$ & $\mathbf{2 0 1 1}$ & Total \\
\hline Kéthiak & 25268,56 & 29371,48 & 31743,6 & 30846,93 & 32288,87 & 34570,3 & $\mathbf{1 8 4} \mathbf{0 8 9 , 7 4}$ \\
Guedj & 3736,35 & 3612,11 & 4594 & 4248,96 & 3525,52 & 4551,85 & $\mathbf{2 4} \mathbf{2 6 8 , 7 9}$ \\
Tambadieng & 3352,42 & 3777,9 & 3352 & 3712,52 & 2928,71 & 3128,26 & $\mathbf{2 0} \mathbf{2 5 1 , 8 1}$ \\
Mérotah & 1220,13 & 2032,31 & 3534,1 & 3490,68 & 6044,04 & 3369 & $\mathbf{1 9 6 9 0 , 2 6}$ \\
Saly & 3342,53 & 2619,9 & 2599,4 & 2751,23 & 2459,78 & 2523,81 & $\mathbf{1 6 2 9 6 , 6 5}$ \\
Yet & 952,42 & 1023,78 & 1040,1 & 1029,53 & 1292,53 & 1251,9 & $\mathbf{6 5 9 0 , 2 6}$ \\
Toufa & 96,59 & 179,15 & 99,8 & 140,06 & 139,49 & 164 & $\mathbf{8 1 9 , 0 9}$ \\
Pagne & 8,06 & 56,52 & 120,3 & 174,58 & 201,61 & 196,42 & $\mathbf{7 5 7 , 4 9}$ \\
Yokhos & 12,57 & 16,09 & 17,5 & 35,36 & 71,37 & 50,9 & $\mathbf{2 0 3 , 7 9}$ \\
Aileron & 46,98 & 26,81 & 22 & 27,03 & 18,27 & 35,3 & $\mathbf{1 7 6 , 3 9}$ \\
Crevette & 1,2 & 27,52 & 24,8 & 17,75 & 24,34 & 28,32 & $\mathbf{1 2 3 , 9 3}$ \\
Autres & 12,14 & 13,2 & 10,9 & 7,92 & 6,48 & 10,56 & $\mathbf{6 1 , 2}$ \\
\hline Total & $\mathbf{3 8 0 4 9 , 9 5}$ & $\mathbf{4 2 7 5 6 , 7 7}$ & $\mathbf{4 7 1 5 8 , 5}$ & $\mathbf{4 6 4 8 2 , 5 5}$ & $\mathbf{4 9 0 0 1 , 0 1}$ & $\mathbf{4 9 8 8 0 , 6 2}$ & $\mathbf{2 7 3 3 2 9 , 4}$ \\
\hline
\end{tabular}

Source: DPM, 2006-2011. Résultats généraux des pêches 
N. G. FALL et al. / Int. J. Biol. Chem. Sci. 8(6): 2523-2538, 2014

Tableau 2 : Espèces de poissons utilisées pour la fabrication du Guedj au Sénégal.

\begin{tabular}{lcc}
\hline Noms vernaculaires & Noms courants & Noms scientifiques \\
\hline Kong & Machoiron & Arius latisculatus \\
Siket Mbao & Faux capitaine & Galeoides decadactylus \\
Ngonkeur & Capitaine ou otolithe & Pseudotolithus brachygnatus \\
Berr & Courbine & Argyrosomus reguis \\
Dorade & Dorade rose & Dentex sp \\
Lagna-lagna & Carangue ou sapater & Chloroscombrus chrysures \\
Gaindé guéthe & Requin & Rhizoprionodon acutus \\
Banda & Dorade grise & Plectorhynchus mediterraneus \\
Sompat & Sompat & Pomadasys jubelini \\
Brochet & Brochet & Sphyraena guachancho \\
\hline
\end{tabular}


N. G. FALL et al. / Int. J. Biol. Chem. Sci. 8(6): 2523-2538, 2014

Tableau 3 : Caractéristiques physico-chimiques et microbiologiques du Guedj ( $n=19)$.

\begin{tabular}{|c|c|c|c|}
\hline & \multicolumn{3}{|c|}{ Guedj collecté des marchés locaux et sites de transformation $(n=19)$} \\
\hline & Min & Moy & Max \\
\hline \multicolumn{4}{|l|}{ Paramètres physico-chimiques } \\
\hline Humidité (\%) & 42,86 & $50,35 \pm 4,49$ & 60,23 \\
\hline Protéines $\left(\mathrm{N}^{*} 6,25\right)(\%)$ & 23,22 & $28,37 \pm 2,50$ & 32,79 \\
\hline Matières grasse $(\%)$ & 0,06 & $2,22 \pm 1,86$ & 6,96 \\
\hline Chlorures de sodium (\%) & 4,9 & $7,9 \pm 1,56$ & 9,6 \\
\hline $\mathrm{pH}$ (solution à $10 \%)$ & 6,05 & $6,28 \pm 0,54$ & 7,12 \\
\hline Histamine $(\mathrm{mg} / 100 \mathrm{~g})$ & & $<0,01$ & \\
\hline \multicolumn{4}{|l|}{ Micro-organismes (UFC/g) } \\
\hline Flore aérobie totale & $3,510^{4}$ & $18,47 \pm 0,24 \quad 10^{4}$ & $9110^{4}$ \\
\hline Coliformes fécaux & & $<10$ & \\
\hline Clostridium sulfito-réducteurs & 10 & $17,64 \pm 2,2 \quad 10^{1}$ & $8010^{1}$ \\
\hline Staphylocoques pathogènes & & $<100$ & \\
\hline Salmonelles & & Absence & \\
\hline Lactobacillus & $0,5210^{2}$ & $2,47 \pm 10^{2}$ & $4,310^{2}$ \\
\hline
\end{tabular}




\section{DISCUSSION}

L'étude a montré que la production du guedj est essentiellement une activité de femmes. Un résultat similaire a été retrouvé par Dème et al. (2003) avec (80\%) de femmes et $20 \%$ d'hommes. L'activité implique quatre acteurs (les pêcheurs, les transformateurs, les commerçants et les consommateurs). Les transformateurs constituent un maillon important et influent beaucoup sur le système de production (nature, qualité et taille de la matière première, qualité et prix du produit fini). Ils sont majoritairement des Sénégalais. Ceci montre le caractère national marqué de la transformation artisanale des produits de la pêche (Dème et al., 2003). La main d'œuvre masculine est majoritairement composée d'étrangers comme rapporté par Kouakou et al. (2013) pour la filière d'adjuevan.

L'activité de production et de commercialisation est très rentable dans son ensemble mais pas de manière équilibrée. Elle est plus rentable pour les grossistes qui disposent de moyen financier et logistique et moindre pour les transformateurs et les détaillants qui subissent les fluctuations de prix engendrées par les grossistes. Cette activité est une source importante de revenus dans les zones rurales, périurbaines et urbaines comme dans le cas du chepa sbutki du Bangladesh (Nayeem et al., 2010). Les revenus varient en fonction de l'importance de la production, des équipements utilisés et de la clientèle. Ils sont généralement utilisés pour régler les dépenses quotidiennes (l'alimentation, les frais de scolarités et soin des enfants, les réparations du matériel de production...).

La majorité des acteurs de la filière sont des illettrés comme également rapporté par Anihouvi et al. (2005) pour la filière du lanhouin. Traditionnellement, il s'agissait d'une activité qui relevait uniquement des femmes issues de familles des zones côtières. Contrairement aux productrices de l'adjuevan dont certaines n'ont aucun lien avec les pêcheurs (Kouakou et al., 2013), (80\%) des productrices de guedj déclarent être filles, sœurs ou épouses de pêcheurs. L'activité de transformation se transmet de génération en génération comme rapporté par Anihouvi et al. (2005) pour le lanhouin. Les femmes la pratiquent depuis la jeunesse et elles ont directement hérité des espaces de travail, du matériel et des savoirs-faire nécessaires de leurs mères qui exerçaient ce métier.

Les techniques de production actuellement utilisées sont traditionnelles et ne permettent pas de présenter un produit de qualité sur le marché. Après analyse des systèmes de production, le risque majeur pour la consommation de ce produit reste d'ordre sanitaire. Les conditions de débarquement, de production, de transport et de conservation généralement médiocres accélèrent la prolifération bactérienne et compromettent la qualité du produit fini.

Deux matières premières sont utilisées pour la fabrication du guedj: le poisson et le sel marin. Le poisson est une denrée très périssable et sa dégradation est en effet la résultante de l'activité in situ des enzymes et des microorganismes notamment des bactéries (Baird, 2000 ; Gram et Dalgaard, 2000 ; Diop et al., 2009). De plus, la préparation du poisson est réalisée dans des conditions environnementales mal adaptées du point de vue sanitaire (Diei-Ouadi, 2005). Or certains microorganismes tels que les entérobactéries qui produisent des amines biogéniques sont responsables de maladies chez l'homme (Olsen et al., 2000). Le sel est utilisé comme seul additif alimentaire pour la fermentation du guedj comme dans le cas du momoni (Sanni et al., 2002). En Asie, la fermentation se fait avec uniquement du sel comme le $Y u$-lu 
en chine ou en complément du sel, un apport de riz, d'ail, de jus de palme comme pour le plaa-som en Thaïlande (Paludan- Müller et al., 2002 ; Jin-Jin et al., 2007). Notons qu'au Sénégal, le processus de production du guedj (salage, fermentation et séchage) ne dure que 8 jours alors qu'en Thaïlande, la fabrication de produits similaires tels que le kapi dure deux mois et celle du plaa-som 12 jours (Paludan- et al., 2002; Nandhsha et al., 2009). Contrairement à la région d'Asie du Sud où la chair du poisson peut être liquéfiée ou transformée en une pâte, au Sénégal comme au Benin et en Côte d'ivoire, après fermentation, la texture du poisson est sous une forme solide (Anihouvi et al., 2005; Kouakou et al., 2013). Les équipements de fortune (claies en bois de rônier, filet de récupération,...) utilisés par les femmes lors du séchage ne permettent pas un bon séchage du produit. En plus, les espaces réduits, entraînent une mauvaise circulation de l'air alors que le séchage du produit nécessite un équipement adéquat en plus d'une bonne circulation de l'air. Or selon Oksuz et al. (2008), le séchage empêche le développement rapide des microorganismes et surtout la poursuite des réactions enzymatiques durant la conservation, modifiant ainsi négativement la qualité organoleptique et sanitaire des produits. La croissance microbienne et les réactions enzymatiques favorisées par l'humidité seraient donc responsables de la destruction du produit fini pouvant entraîner d'importantes pertes économiques. Ainsi, le poisson est exposé à divers prédateurs et peut être contaminé par les insectes ichtyophages et les larves de lucilie. Les mesures prises par les femmes pour faire face à cette situation sont l'utilisation de manière abusive de produits chimiques (dose non respectée) sur les produits finis les rendant parfois impropres à l'alimentation humaine (intoxication alimentaire). Le manque d'équipements adéquats et la vétusté du matériel pour le conditionnement, l'emballage, la conservation et le transport pourraient être sources potentielles de contamination microbienne et chimique.

Par ailleurs, les résultats obtenus de la caractérisation du Guedj montrent une grande variation des paramètres chimiques et microbiologiques d'un produit à l'autre selon les sites de transformation et de vente. Cette dispersion des résultats d'analyse se justifie entre autres, par une différence du niveau d'hygiène des sites de production et de vente. Les données physiques (humidité relative) et chimique (salinité) édifient sur les conditions de stockage et de conservation des produits finis. Le $\mathrm{pH}$ est environ 6-7, des valeurs similaires de $\mathrm{pH}$ ont été rapportées par Anihouvi et al. (2005) pour le lanhouin et Sanni et al. (2002) pour le momoni. L'analyse microbiologique montre une présence élevée de microorganismes aérobies, avec une moyenne de $18,4710^{4}$ ufc. $^{-1}$. Ce résultat, comparé à ceux trouvés par (Kouakou et al., 2013; Anihouvi et al., 2006) (4,8 $10^{8}$ et6,5 $10^{5}$ ) (ufc $\mathrm{g}^{-1}$ ) montre un niveau de contamination plus faible pour le guedj. Ainsi, sur le plan sanitaire, on note une absence de salmonelle dans tous les échantillons, cependant la présence de clostridies comme trouvé par Kouakou et al. (2013) pour l'adjuevan montre que le guedj est aussi une source de contamination.

\section{Conclusion}

Il ressort de cette étude que l'activité de transformation du poisson fermenté et séché est très importante au vu des capacités de production, de commercialisation et des revenus générés aux acteurs. Cependant, ce segment de la filière poisson connaît beaucoup de difficultés liées à la manutention, 
aux techniques de transformation rudimentaires utilisées, au manque d'infrastructures mais aussi aux conditions d'hygiène déplorables. La qualité du produit n'est ni régulière ni contrôlée, ce qui entraîne une baisse de la valeur nutritionnelle du produit. Les difficultés de stockage contribuent en partie aux pertes estimées entre 25 et $30 \%$.

Pour lever ces contraintes, il y a nécessité de former et sensibiliser les acteurs aux bonnes pratiques d'hygiène et de fabrication (BPH et BPF), à l'utilisation de nouvelles technologies. Par ailleurs, la mise au point d'un starter microbien pour garantir une fermentation contrôlée pourrait influer positivement sur la qualité sanitaire et organoleptique du produit fini.

\section{REMERCIEMENTS}

Les auteurs remercient le West African Agriculture Productivity Program (WAAPP) de la Banque Mondiale, le centre d'Etude Régional pour l'Amélioration de l'adaptation à la Sécheresse (CERAAS /ISRA), l'Institut de Technologie Alimentaire (ITA), l'Institut Universitaire de Pêche et d'Aquaculture (IUPA) et l'Université de Liège à travers son Centre Wallon de Biologie Industrielle (CWBI/ULg) pour avoir mis à la disposition les ressources matérielles, financières et humaines nécessaires à la mise en œuvre de cette étude.

\section{REFERENCES}

AFNOR. 2002. Méthode de Routine et d'Evaluation, Microbiologie Alimentaire (tome 2, $8^{\text {éme }}$ édn). AFNOR : Paris ; 269.

Anihouvi VB, Hounhouigan JH, Ayernor GS. 2005. La production et la commercialisation dulanhouin, un condiment à base de poisson fermenté du Golfe du Bénin. Cahier Agri., 14(3): 323-330.

Anihouvi VB, Ayernor GS, Hounhouigan JD, Sakyi Dawson E. 2006. Quality characteristics of lanhouin: a traditionally processed fermented fish product in the republic of Benin, p.15.

AOAC (Association of Official Analytical Chemists). 2007. Official Methods of Analysis (vol 2, $18^{\text {th }}$ edn). AOAC.

Baird-Parker TC. 2000. The production of microbiologically safe and stable foods. In The Microbiological Safety and Quality of Foods, Lund BM, Baird Parker TC, Gould GW (eds). Aspen Publishers: Gaisther-burg; 2000.

Déme M, Thiam D, BA M. 2003. Recensement des femmes transformatrices de la pêche artisanale sénégalaise. Doc. CRODT/PAPASUD, p.170.

Diei-Ouadi Y. 2005. Minced sardinella fillets in fish-landing and marketing sites in Senegal. FAO fisheries circular No 999 : FIIU/C999 (EN). Rome: FAO

Diop M, Destain J, Tine E, Thonart P. 2009. Les produits de la mer au Sénégal et le potentiel des bactéries lactiques et des bactériocines pour la conservation. Biotechnol. Agron. Soc. Environ., 14(2): 341-350.

DPM (Direction des Pêches Maritimes). 20062011. Résultats généraux de la pêche maritime sénégalaise. Rapports 20062011, Dakar.

Gram L, Dalgaard P. 2002. Fish spoilage bacteria problems and solutions. Curr. Opin. Biotechnol., 13: 262-266.

Hubert A. 2003. Des aliments fermentés en Asie du Sud-Est et du poisson en particulier : les fermentations au service des produits du terroir. 
Colloque INRA/INAO Paris, 8 octobre 2003.

Hubert A, Sabban F. 2005. Des produits traditionnels en en Asie du Sud_Est : Des aliments fermentés et des poissons en particulier: les fermentations au service des produits du terroir. Colloque INRA/INAO Paris, 2005.

Jin-Jin J, Qing-Xiao Z, Zhi-Wei Z, Li-Yan Z. 2007.Chemical and sensory changes associated $\mathrm{Yu}$-lu fermentation process A traditional Chinese fishsauce. Food Chemistry, 104: 1629-1634.

Kopermsub P, Yunchalard S. 2010. Identification of lactic and acid bacteria associated with the production of plaasom, a traditional fermented fish product of Thailand. International Journal of Food Microbiology, 138: 200-204

Kouakou AC, Kouadio FNG, Dadie AT, Montet D, Djè MK. 2013. Production et commercialisation de l'adjuevan, poisson fermenté de Côte d'Ivoire. Cah. Agric., 22 : 559-567.

Mbaye L. 2005. Etat des lieux de la filière de transformation artisanale des produits halieutiques au Sénégal, Doc. Gret, Enda Graf Sahel, p. 40.

Nandhsha F, Soottawat B, Suttirug P, Wanwisa B. 2009. Chemical composition and antioxidative activity of Thai traditional fermented shrimp and krill products. Journal of Food Chemistry, 119: 133-140.

Nayeem M, Pervi K, Reza M, Khan M, Islam M, Kamal M. 2010. Marketing System of traditional dried and semi-fermented fish product (cheap shutki) and socioéconomic condition of the retailers in local market of Mynensingh region Bangladesh. Bangladesh Research Publication Journal, 4: 69-75.

Oksuz A, Evrendilek G, Calis M, Orezen A. 2008. Production of a dry sausage from African fish culture. Journal of Aquaculture in the Tropics, 5: 87-90.

Olsen.SJ, Mackinon LC, Goulding JS, Bean NH, Slutsker L. 2000. Surveillance for foodborne-desease outbreaks-United States, 1993-1997.Morbidity Weekly report CDC. Surveill Summ., 49: 1-62.

Paludan-Müller C, Madsen M, Sophanodora P, Gram L, Mommer P. 2002. Fermentation and microflora of plaasom, a thai fermented fish product prepared with different salt concentrations. International Journal of Food Microbiology, 73: 61-70.

Sanni A, Asiedu M, Ayernor G. 2002. Microflora and chemical composition of momoni, a Ghanaian fermented fish condiment. Journal of Food Composition and Analysis, 15: 577583. 